Pure and Applied Mathematics Quarterly

Volume 7, Number 4

(Special Issue:

In memory of Eckart Viehweg)

1297-1307, 2011

\title{
The Hodge Bundle on Hurwitz Spaces
}

Gerard van der Geer and Alexis Kouvidakis

In memoriam Eckart Viehweg

\begin{abstract}
In 2009 Kokotov, Korotkin and Zograf gave in [7] a formula for the class of the Hodge bundle on the Hurwitz space of admissible covers of genus $g$ and degree $d$ of the projective line. They gave an analytic proof of it. In this note we give an algebraic proof and an extension of the result.
\end{abstract}

Keywords: Hurwitz space, Hodge class, moduli of curves.

\section{INTRODUCTION}

Let $\mathcal{H}_{g, d}$ be the Hurwitz space of degree $d$ covers of $\mathbb{P}^{1}$ of genus $g$ and with simple branch points. It parametrizes covers $f: C \rightarrow \mathbb{P}^{1}$ with $C$ irreducible, smooth of genus $g$ and $f$ of degree $d$ with $b=2 g-2+2 d$ simple branch points that are marked. This space admits a compactification $\overline{\mathcal{H}}_{g, d}$, the space of admissible covers of genus $g$ and degree $d$; that is, covers $f: C \rightarrow P$, where $C$ is a nodal curve, $P$ is a stable $b$-pointed curve of genus 0 and $f$ an admissible cover in the sense of [5], see also [4].

The Hurwitz space $\overline{\mathcal{H}}_{g, d}$ is a coarse moduli space, but it is not smooth, not even normal. The boundary $\overline{\mathcal{H}}_{g, d}-\mathcal{H}_{g, d}$ consists of finitely many divisors $\Delta_{k, \mu}=$ $\Delta_{b-k, \mu}$ indexed by a partition $b=k+(b-k)$ with $2 \leq k \leq b-2$ and a 'format' $\mu=\left(m_{1}, \ldots, m_{r}\right)$ where the $m_{i}$ are natural numbers with $\sum_{i=1}^{r} m_{i}=d$. Note that $\Delta_{k, \mu}$ will in general be reducible. A generic point in $\Delta_{k, \mu}$ corresponds to the

Received: Oct. 15, 2010.

1991 Mathematics Subject Classification. 14C25,14H40. 
case where $P$ is a curve of genus 0 with two components $P_{1}$ and $P_{2}$ intersecting in one point $Q$, with $P_{1}$ having $k$ branch points, and the inverse image of $Q$ consists of $r$ points $Q_{1}, \ldots, Q_{r}$ with ramification indices $m_{1}, \ldots, m_{r}$.

There are natural maps $q: \overline{\mathcal{H}}_{g, d} \rightarrow \overline{\mathcal{M}}_{0, b}$ to the moduli space $\overline{\mathcal{M}}_{0, b}$ of stable $b$-pointed curves of genus 0 , and $\varphi: \overline{\mathcal{H}}_{g, d} \rightarrow \overline{\mathcal{M}}_{g}$ to the moduli space $\overline{\mathcal{M}}_{g}$ of stable curves of genus $g$. These are defined by assigning to $f: C \rightarrow P$ the stable curve $P$, resp. the stabilized model of $C$.

The Hurwitz space $\overline{\mathcal{H}}_{g, d}$ carries a natural $\mathbb{Q}$-divisor class, the Hodge class $\lambda$. It is the pullback of the first Chern class of the Hodge bundle on $\overline{\mathcal{M}}_{g}$. The boundary divisors $\Delta_{k, \mu}$ define $\mathbb{Q}$-divisor classes which are taken in the orbifold sense, that is, counted with a weight $1 / a$ where $a$ is the order of the automorphism group of an object corresponding to the generic point of the boundary divisor, see Section 3.

The theorem of Kokotov, Korotkin and Zograf expresses the Hodge class $\lambda$ on $\overline{\mathcal{H}}_{g, d}$ in terms of the boundary divisor classes. In terms of the Picard group of the corresponding functor (or stack) the theorem reads

Theorem 1.1. The Hodge class $\lambda$ of the functor $\overline{\mathcal{H}}_{g, d}$ is given by

$$
\lambda=\sum_{k=2}^{b / 2} m(\mu)\left(\frac{k(b-k)}{8(b-1)}-\frac{1}{12}\left(d-\sum_{i=1}^{r} \frac{1}{m_{i}}\right)\right) \delta_{k, \mu}
$$

where $b=2 g+2 d-2$ and $\delta_{k, \mu}$ is the class of $\Delta_{k, \mu}$ and $m(\mu)$ is the least common multiple of $m_{1}, \ldots, m_{r}$.

Kokotov, Korotkin and Zograf used anaytic tools, esp. the tau-function, to construct a trivializing section of the Hodge bundle on $\mathcal{H}_{g, d}$ and calculated the vanishing orders of this section along the boundary divisors. In our approach we shall apply Grothendieck-Riemann-Roch to the relative dualizing sheaf on the admissible cover $f: C \rightarrow P$ over a base $S$ with structure map $t: C \rightarrow S$; this will provide us with an expression of $\lambda$ in terms of $t_{*} \omega_{t}^{2}$ and boundary divisors and then we will express $t_{*} \omega_{t}^{2}$ too in terms of boundary divisors. In fact, if $f: C / S \rightarrow P / S$ is any family of admissible covers of genus $g$ and degree $d$ then 
we have a commutative diagram

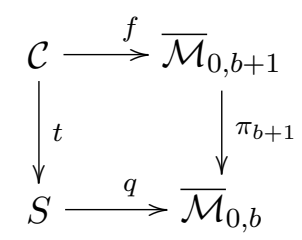

The relative dualizing sheaf $\omega_{t}$ of $t$ can be expressed in terms of the ramification divisor $R$ of the map $f$ and the pullback of the relative dualizing sheaf $\omega_{\pi_{b+1}}$ of $\pi_{b+1}$. Working this out will give us our proof.

This proof extends also to other cases. As an example we consider the Hurwitz space $\mathcal{H}_{g, d, l}$ of covers $f: C \rightarrow P$ with $C$ smooth of genus $g$ and $P=\left(\mathbb{P}^{1}, p_{1}, \ldots, p_{b}\right)$ a $b$-pointed smooth curve of genus $0, f$ a morphism of degree $d$ with simple branch points $p_{i}$ for $i=2, \ldots, b$ and one branch point $p_{1}$, over which $f$ is étale except for one $l$-fold ramification point. Note that $2 g=b-2 d+l$. The dimension of this space is $2 g+2 d-l-3$. This space admits a compactification $\overline{\mathcal{H}}_{g, d, l}$ by so-called $l$-admissible covers where the curve $f: C \rightarrow P$ satisfies the axioms for admissibility except over $p_{1}$ over which we have exactly one ramification point of degree $l$, cf. [3], where this Hurwitz space was introduced. Then the expression for $\lambda$ on the functor (or stack) is the following.

Theorem 1.2. The Hodge class of the functor $\overline{\mathcal{H}}_{g, d, l}$ is given by

$$
\begin{aligned}
\lambda=\sum_{k=2}^{[b / 2]} \sum_{\mu} m(\mu)\left(\frac{k(b-k)}{8(b-1)}-\frac{1}{12}\left(d-\sum_{i=1}^{r} \frac{1}{m_{i}}\right)\right) \delta_{k, \mu} \\
\quad+\frac{(2 l+1)(l-2)}{24 l} \sum_{k=2}^{b-2} \sum_{\mu} m(\mu) \frac{(b-k)(b-1-k)}{(b-1)(b-2)} \delta_{k, \mu}^{1},
\end{aligned}
$$

where $b=2 g+2 d-l$ and $\delta_{k, \mu}^{1}$ the part of $\delta_{k, \mu}$ with general member an admissible cover that maps to a b-pointed curve whose component with the $k$ marked points contains the point $p_{1}$.

In [2] we gave an application of the formula of Kokotov, Korotkin and Zograf by calculating an important divisor class on $\overline{\mathcal{M}}_{g}$ for $g$ even. 


\section{Divisors ON $\overline{\mathcal{M}}_{0, b}$}

We recall some basic facts about the divisor theory of $\overline{\mathcal{M}}_{0, b}$, see [6]. The boundary of $\overline{\mathcal{M}}_{0, b}$ is the union of irreducible divisors, each of which corresponds to a decomposition of $B=\{1, \ldots, b\}$ as $B=\Lambda \sqcup \Lambda^{c}$ into two disjoint subsets with $2 \leq \# \Lambda \leq b-2$. We write the corresponding divisor as $S_{b}^{\Lambda}$ modulo the relation $S_{b}^{\Lambda}=S_{b}^{\Lambda^{c}}$. If one wishes one can normalize the $\Lambda$ by requiring that

$$
\#(\Lambda \cap\{1,2,3\}) \leq 1
$$

With $\Lambda \subset\{1, \ldots, b\}$, the generic element of the divisor $S_{b}^{\Lambda}$ represents a stable curve with two rational components, with the marked points of $\Lambda$ on one component. The map $\pi_{b+1}: \overline{\mathcal{M}}_{0, b+1} \rightarrow \overline{\mathcal{M}}_{0, b}$ is equipped with $b$ sections $s_{i}: \overline{\mathcal{M}}_{0, b} \rightarrow$ $\overline{\mathcal{M}}_{0, b+1}$ with $i=1, \ldots, b$. We can interpret $\bar{M}_{0, b+1}$ as the universal curve over $\bar{M}_{0, b}$.

The boundary divisors of $\overline{\mathcal{M}}_{0, b+1}$ are related to those of $\overline{\mathcal{M}}_{0, b}$ as follows:

$$
\pi_{b+1}^{*} S_{b}^{\Lambda}=S_{b+1}^{\Lambda} \cup S_{b+1}^{\Lambda \cup\{b+1\}},
$$

with $\Lambda \subset\{1, \ldots, b\}$. Note that if $\Lambda \subset\{1, \ldots, b\}$ is normalized, then so are $\Lambda$ and $\Lambda \cup\{b+1\}$ as subsets of $\{1, \ldots, b+1\}$. So all the boundary components of $\overline{\mathcal{M}}_{0, b+1}$ are coming from $\overline{\mathcal{M}}_{0, b}$ except the components $S_{b+1}^{\{i, b+1\}}(i=1, \ldots, b)$ that correspond to the image of the $b$ sections $s_{i}$. The map $S_{b+1}^{\Lambda} \rightarrow S_{b}^{\Lambda}$ (resp. $\left.S_{b+1}^{\Lambda \cup\{b+1\}} \rightarrow S_{b}^{\Lambda}\right)$ is generically a $\mathbb{P}^{1}$-fibration.

Recall that $B=\{1, \ldots, b\}$. In $\overline{\mathcal{M}}_{0, b}$ we define for $2 \leq j<b / 2$ the divisors

$$
T_{b}^{j}=\sum_{\Lambda \subset B, \# \Lambda=j} S_{b}^{\Lambda} \quad \text { and if } b \text { is even } \quad T_{b}^{b / 2}=\frac{1}{2} \sum_{\Lambda \subset B, \# \Lambda=b / 2} S_{b}^{\Lambda} .
$$

On the moduli space $\overline{\mathcal{M}}_{0, b}$ we have the tautological classes $\psi_{i}$ for $1 \leq i \leq b$, defined as the first Chern class of the line bundle that associates to a pointed curve $\left(C, p_{1}, \ldots, p_{b}\right)$ the cotangent space to $C$ at $p_{i}$. We put $\psi=\sum_{i=1}^{b} \psi_{i}$.

On $\overline{\mathcal{M}}_{0, b}$ we have the relation

$$
\psi_{i}=\sum_{j=1}^{b-3} \frac{(b-1-j)(b-2-j)}{(b-1)(b-2)} \sum_{A \subset\{1, \ldots, b\}-\{i\}, \# A=j} S_{b}^{\{i\} \cup A},
$$


cf. [1], Lemma 1 on page 1186. Therefore we get

$$
\psi:=\sum_{i=1}^{b} \psi_{i}=\sum_{j=2}^{[b / 2]} \frac{(b-j) j}{b-1} T_{b}^{j} .
$$

\section{Divisors on the Hurwitz Space}

In this section we are concerned with divisors on our Hurwitz space. Recall that the boundary of $\overline{\mathcal{H}}_{g, d}$ consists of finitely many divisors $\Delta_{k, \mu}=\Delta_{b-k, \mu}$, where for each irreducible component of $\Delta_{k, \mu}$ the generic point corresponds to an admissible cover $f: C \rightarrow P$ of degree $d$ with $P$ a stable $b$-pointed genus 0 curve consisting of two copies $P_{i}(i=1,2)$ of $\mathbb{P}^{1}$ with one intersection point $Q$ and with $k$ marked points on $P_{1}$ and with ramification points $Q_{i}$ of ramification degree $m_{i}(i=1, \ldots, r)$ over $Q$. One can decompose these divisors further as $\Delta_{k, \mu}=\sum \Delta_{\Lambda, \mu}$ corresponding to decompositions $\{1, \ldots, b\}=\Lambda \sqcup \Lambda^{c}$.

We start by giving a local description of admissible covers, cf. the discussion in Harris-Mumford [5], p. 61-62. We then have the diagram

$$
\begin{array}{r}
\overline{\mathcal{M}}_{0, b+1} \\
\bar{H}_{g, d} \stackrel{q}{\longrightarrow} \bar{\vee}^{\pi_{b+1}}
\end{array}
$$

We now take a general point $\gamma$ of an irreducible component of $\Delta_{k, \mu}$. It corresponds to a general admissible cover $f: C \rightarrow P$ with $P$ a stable genus 0 curve with two components $P_{1}$ and $P_{2}$ intersecting transversally in one point $Q$. The point $\gamma$ maps under $q$ to the point of $S_{b}^{\Lambda}$ determined by $P$. If $Q_{1}, \ldots, Q_{r}$ are the preimages of $Q$ under $f$ with ramification format $\mu=\left(m_{1}, \ldots, m_{r}\right)$ then locally near $Q_{i}$ the curve $C$ is given by $x_{i} y_{i}=s_{i}$ and locally near $Q$ the curve $P$ is given by $u v=t_{1}$ with $u=x_{i}^{m_{i}}$ and $v=y_{i}^{m_{i}}$. By [5] the formal neighborhood of $\gamma$ that pro-represents infinitesimal deformations is given by $\operatorname{Spec}(R)$ with

$$
R=\mathbb{C}\left[t_{1}, t_{2}, \ldots, t_{b-3}, s_{1}, \ldots, s_{r}\right] /\left\langle s_{j}^{m_{j}}=t_{1}, j=1, \ldots, r\right\rangle .
$$

We will use the notation

$$
m(\mu)=\text { least common multiple of } m_{1}, \ldots, m_{r} .
$$


The following fact is known; for the reader's convenience we give a proof.

Lemma 3.1. The normalization of $\overline{\mathcal{H}}_{g, d}$ has $m_{1} \cdots m_{r} / m(\mu)$ branches $E_{i}$ along any irreducible component of $\Delta_{k, \mu}$ and the ramification degree of the map $E_{i} \rightarrow$ $\overline{\mathcal{M}}_{0, b}$ is $m(\mu)$.

Proof. To normalize we introduce the local parameter $\tau=t_{1}^{1 / m(\mu)}$ and write $s_{j}=\zeta_{m_{j}} \tau^{m(\mu) / m_{j}}$ with $\zeta_{m_{j}}$ an $m_{j}$ th root of unity. This will give a normalization. By letting the $m(\mu)$ th roots of unity act via reparametrizations $\tau \mapsto \zeta_{m(\mu)} \tau$ we see that $\mu(m)$ solutions give the same branch, leading to $m_{1} \cdots m_{r} / m(\mu)$ branches. For each of the branches the ramification index is $m(\mu)$.

We want to prove a relation in the rational Picard group $\operatorname{Pic}_{\mathbb{Q}}\left(\bar{H}_{g, d}\right)$. Unfortunately, this group is not known, but it is conjectured that it is generated by boundary classes, cf. [4], p. 66, Conj. 2.49 .

We let $\lambda$ be the Hodge class on $\overline{\mathcal{H}}_{g, d}$. It is defined functorially by taking for each admissible cover

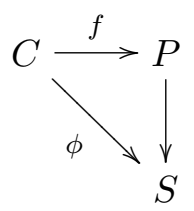

the first Chern class of $\phi_{*} \omega_{C / S}$ with $\omega_{C / S}$ the relative dualizing sheaf.

The divisors that we use will be viewed in the orbifold sense; that is, we will weight an irreducible divisor $D$ with a factor $1 / A$ where $A$ is the order of the automorphism group of the object corresponding to the generic point of $D$. So in a relation $\lambda=\sum c_{k, \mu} \delta_{k, \mu}$, like the one of Theorem 1.1, the class $\delta_{k, \mu}$ is the sum of the classes of the irreducible components of $\Delta_{k, \mu}$ weighted by 1 over the order of the automorphism group of the object corresponding to the generic point of the component.

In order to prove the relation we first work on the normalization $\tilde{\mathcal{H}}_{g, d}$ of $\overline{\mathcal{H}}_{g, d}$. To prove a relation $\tilde{\lambda}=\sum \tilde{c}_{k, \mu} \tilde{\delta}_{k, \mu}$ there it suffices to prove for every family of admissible covers over a smooth 1-dimensional base $S$ not contained in the boundary of our Hurwitz space, a relation $\lambda_{S}=\sum \tilde{c}_{k, \mu} \delta_{k, \mu}^{\prime}$ with $\lambda_{S}$ and $\delta_{k, \mu}^{\prime}$ the pullbacks under the classifying maps $S \rightarrow \tilde{H}_{g, d}$, cf. the discussion on p. 141-146 of [4]. Then if we descend from the normalization $\tilde{\mathcal{H}}_{g, d}$ of $\overline{\mathcal{H}}_{g, d}$ to the space $\overline{\mathcal{H}}_{g, d}$ itself by the normalization map $\nu: \tilde{\mathcal{H}}_{g, d} \rightarrow \overline{\mathcal{H}}_{g, d}$ we have to take into account 
that the pushforward of a boundary component $\tilde{\Delta}_{k, \mu}$ on $\tilde{\mathcal{H}}_{g, d}$ results in a multiple (cf. Lemma 3.1) of the (reduced) cycle $\Delta_{k, \mu}$ on $\overline{\mathcal{H}}_{g, d}$, viz.

$$
\nu_{*}\left[\tilde{\Delta}_{k, \mu}\right]=\frac{m_{1} \cdots m_{r}}{m(\mu)}\left[\Delta_{k, \mu}\right],
$$

and this gives rise to a factor $m_{1} \cdots m_{r} / m(\mu)$ in the coefficients $c_{k, \mu}$.

Using now 1-dimensional smooth families of admissible curves we now prove that $\lambda$ is a pullback from $\overline{\mathcal{M}}_{g}$.

Lemma 3.2. The Hodge bundle on $\overline{\mathcal{H}}_{g, d}$ is the pullback under the natural map $\varphi: \overline{\mathcal{H}}_{g, d} \rightarrow \overline{\mathcal{M}}_{g}$ of the Hodge bundle on $\overline{\mathcal{M}}_{g}$.

Proof. Given such a family $f: C \rightarrow P / S$ of admissible covers we desingularize the total space of $C$ to get $f^{\prime}: C^{\prime} \rightarrow S$. This does not affect the Hodge bundle, see e.g. [4], p. 156. Then we stabilize $C^{\prime}$ to get a family $X$ of stable curves over $S$. This is done step by step by first contracting exceptional curves in fibres of $f^{\prime}$, say $\alpha: C^{\prime} \rightarrow X$ is a contraction. But this does not change the Hodge bundle: one has $\alpha^{*} \lambda_{X}=\lambda_{C^{\prime}}$; indeed, the degree of $\omega_{C^{\prime}}$ on an exceptional curve is negative, hence the pullback induces an isomorphism $H^{0}\left(X_{s}, \omega_{X}\right) \cong H^{0}\left(C_{s}^{\prime}, \omega_{C^{\prime}}\right)$. In this way we arrive at a model $X^{\prime}$ over $S$; we then have to contract the $(-2)$-configurations in the fibres to get the family of stable curves $X / S$. As remarked above this does not change the relative dualizing sheaf: if $\beta: X^{\prime} \rightarrow X$ is the contraction then $\beta^{*} \omega_{X}=\omega_{X}^{\prime}$.

\section{The Proof}

Let $f: C / S \rightarrow P / S$ be any family of admissible covers of genus $g$ and degree $d$. Since $\overline{\mathcal{M}}_{0, b+1}$ is the universal curve over $\overline{\mathcal{M}}_{0, b}$ we have a commutative diagram

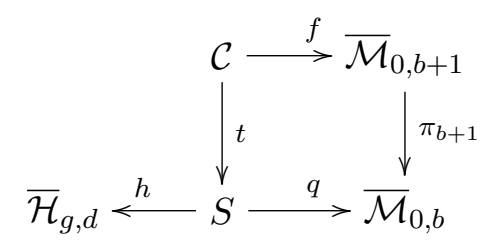

Interpreting the boundary divisor and taking into account the ramification degree of the normalization (see Lemma 3.1 and its proof) we obtain the following.

Lemma 4.1. We have $q^{*}\left(T_{b}^{j}\right)=\sum_{\mu} m(\mu) h^{*} \delta_{j, \mu}$. 
Hereafter we just shall write $\delta_{j, \mu}$ for $h^{*}\left(\delta_{j, \mu}\right)$ on $S$. This is the divisor class on $S$ given by the singular curves of type $(j, \mu)$.

The families $C / S$ and $P / S$ have relative dualizing line bundles $\omega_{t}$ and $\omega_{\pi_{b+1}}$. We let $R$ be the closure of the ramification locus of the map $f$ restricted to the open part of $S$ which is the pullback under $S \rightarrow \mathcal{H}_{g, d}$. Under $f$ this maps to the $b$ sections of the map $\pi_{b+1}$. In view of the diagram (3) we have

$$
\omega_{t}=f^{*} \omega_{\pi_{b+1}}+R
$$

Now $R$ splits as a sum $\sum_{i=1}^{b} R_{i}$ with $R_{i}$ the component of $R$ mapping to the $i$ th section $S_{b+1}^{\{i, b+1\}}$. Since the branching is simple we thus obtain $b$ sections $\tau_{i}: S \rightarrow \mathcal{C}$ of the map $t$.

Lemma 4.2. We have $t_{*}\left(f^{*} \omega_{\pi_{b+1}} \cdot R\right)=q^{*} \psi$ and $t_{*}\left(R^{2}\right)=-(1 / 2) q^{*} \psi$.

Proof. We calculate

$$
\begin{aligned}
t_{*}\left(f^{*} \omega_{\pi_{b+1}} \cdot R\right) & =\sum_{i=1}^{b} t_{*}\left(f^{*} \omega_{\pi_{b+1}} \cdot R_{i}\right)=\sum_{i=1}^{b} t_{*}\left(\tau_{i *} \tau_{i}^{*} f^{*} \omega_{\pi_{b+1}}\right) \\
& =\sum_{i=1}^{b} q^{*} s_{i}^{*} \omega_{\pi_{b+1}}=q^{*}\left(\sum_{i=1}^{b} \psi_{i}\right)=q^{*} \psi
\end{aligned}
$$

where we used $f \circ \tau_{i}=s_{i} \circ q$ and $t \circ \tau_{i}=\mathrm{id}_{S}$. This proves the first claim.

In order to calculate $t_{*}\left(R^{2}\right)$ we observe that the $R_{i}$ are disjoint, hence $R^{2}=$ $\sum R_{i}^{2}$. If we write $f^{*} S_{b+1}^{\{i, b+1\}}=2 R_{i}+A_{i}$ with $A_{i} \cdot R_{i}=0$, we have

$$
R_{i}^{2}=\frac{1}{2}\left(f^{*} S_{b+1}^{\{i, b+1\}}-A_{i}\right) \cdot R_{i}=\frac{1}{2} f^{*} S_{b+1}^{\{i, b+1\}} \cdot R_{i} .
$$

Now we use the adjunction formula to observe that $s_{i}^{*} S_{b+1}^{\{i, b+1\}}=-\psi_{i}$. So we see

$$
\begin{aligned}
t_{*}\left(R^{2}\right) & =\frac{1}{2} \sum_{i=1}^{b} t_{*}\left(f^{*} S_{b+1}^{\{i, b+1\}} \cdot R_{i}\right)=\frac{1}{2} \sum_{i=1}^{b} t_{*} \tau_{i *} \tau_{i}^{*} f^{*} S_{b+1}^{\{i, b+1\}} \\
& =\frac{1}{2} \sum_{i=1}^{b} q^{*} s_{i}^{*} S_{b+1}^{\{i, b+1\}}=-\frac{1}{2} q^{*} \sum_{i=1}^{b} \psi_{i}=-\frac{1}{2} q^{*} \psi .
\end{aligned}
$$

Lemma 4.3. We have $t_{*} f^{*}\left(\omega_{\pi_{b+1}}^{2}\right)=-d \sum_{j=2}^{b / 2} q^{*} T_{b}^{j}$. 
Proof. We write $\hat{S}=\sum_{i=1}^{b} S_{b+1}^{\{i, b+1\}}$ for the sum of the sections. Recall from [1] (Section 2) the identity

$$
\pi_{b+1 *}\left(\left[\omega_{\pi_{b+1}}(\hat{S})\right]^{2}\right)=\kappa_{1}\left(\overline{\mathcal{M}}_{0, b}\right)=\psi-\sum T_{b}^{j} .
$$

If $\kappa=\omega_{\pi_{b+1}} \cdot \hat{S}$ then $\pi_{b+1 *}(\kappa)=\psi$. Indeed, $\pi_{b+1 *}(\kappa)=\sum_{i} \pi_{b+1 *} s_{i *} s_{i}^{*} \omega_{\pi_{b+1}}=$ $\sum_{i} s_{i}^{*} \omega_{\pi_{b+1}}=\sum_{i} \psi_{i}=\psi$. Moreover, by the adjunction formula we have $\pi_{b+1 *}\left(\hat{S}^{2}\right)=$ $-\psi$. Therefore we get

$$
\psi-\sum_{j=2}^{b / 2} T_{b}^{j}=\pi_{b+1 *}\left(\kappa^{2}\right)=\pi_{b+1 *}\left(\omega_{\pi_{b+1}}^{2}+2 \omega_{\pi_{b+1}} \cdot \hat{S}+\hat{S}^{2}\right)=\pi_{b+1 *}\left[\omega_{\pi_{b+1}}^{2}\right]+\psi,
$$

hence $\pi_{b+1 *}\left[\omega_{\pi_{b+1}}^{2}\right]=-\sum_{j=2}^{b / 2} T_{b}^{j}$. Now using $t_{*} f^{*}\left[\omega_{\pi_{b+1}}^{2}\right]=d q^{*} \pi_{b+1 *}\left[\omega_{\pi_{b+1}}^{2}\right]$ we get the required result.

Proposition 4.4. We have the identity

$$
t_{*}\left[\omega_{t}^{2}\right]=\sum_{j=2}^{b / 2}\left(-d+\frac{3}{2} \frac{j(b-j)}{b-1}\right) \sum_{\mu} m(\mu) \delta_{j, \mu} .
$$

Proof. We have $t_{*}\left[\omega_{t}^{2}\right]=t_{*}\left(f^{*} \omega_{\pi_{b+1}}^{2}+2 f^{*} \omega_{\pi_{b+1}} \cdot R+R^{2}\right)$ and the result now follows from lemmas 4.2 and 4.3 and the formula for $\psi$ given in (2).

We now prove Theorem 1.1. In [8] Mumford proved the identity $12 \lambda=\kappa_{1}+\delta$ on the moduli space $\overline{\mathcal{M}}_{g}$. The same approach, applying Grothendieck-RiemannRoch to the morphism $t$ and the relative dualizing sheaf, works for our admissible curve $C / S$ and the proof of Theorem 5.10 of loc. cit. can be transferred almost verbatim (taking into account the arguments in the proof of Lemma 3.2) to give the identity

$$
12 \lambda=t_{*}\left[\omega_{t}^{2}\right]+\sum_{j, \mu} m(\mu) \sum_{i=1}^{r} \frac{1}{m_{i}} \delta_{j, \mu} .
$$

on $S$. Indeed, to get the multiplicity of $\delta_{j, \mu}$ we observe the following. Let $f$ : $C / S \rightarrow P / S$ be a family of admissible covers over a smooth 1-dimensional and such that the general fibre is a smooth curve. We may assume that the image of $S$ in $\overline{\mathcal{H}}_{g, d}$ intersects the divisors $\Delta_{j, \mu}$ only in sufficiently general points (omitting codimension 2 loci where boundary divisors intersect). Let $s \in S$ correspond to an admissible cover $C_{s} \rightarrow P_{s}$ with $P_{s}$ consisting of two components meeting in $Q$ with local equation $u v=t_{1}$ and $Q_{1}, \ldots, Q_{r}$ the points lying over $Q$ with ramification format $\mu=\left(m_{1}, \ldots, m_{r}\right)$ and with local equations $x_{i} y_{i}=s_{i}$ and 
$s_{i}^{m_{i}}=t_{1}$ and $u=x_{i}^{m_{i}}, v=y_{i}^{m_{i}}$. Then when we pull back to $S$ we have because the normalization map on $\overline{\mathcal{H}}_{g, d}$ is ramified of degree $m(\mu)$, a change of coordinates $t_{1}=\sigma^{m(\mu)}$ which leads to local equations around $Q_{i}$ of the form $x_{i} y_{i}=\sigma^{m(\mu) / m_{i}}$. Therefore the contribution of the $Q_{i}$ 's to the singularity locus is $m(\mu) \sum_{i=1}^{r} 1 / m_{i}$. Substituting the expression for $t_{*}\left[\omega_{t}^{2}\right]$ from Proposition 4.4 in equation (4) yields the result.

The proof of Theorem 1.2 goes in a similar fashion. We consider a diagram

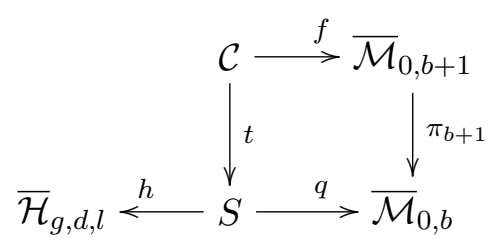

The analogue of Lemma 4.1 holds and we have the formula

$$
\omega_{t}=f^{*} \omega_{\pi_{b+1}}+(l-1) R_{1}+\sum_{i=2}^{b} R_{i}=f^{*} \omega_{\pi_{b+1}}+(l-2) R_{1}+R .
$$

The analogue of Lemma 4.2 says that $t_{*}\left(f^{*} \omega_{\pi_{b+1}} \cdot R_{i}\right)=q^{*} \psi_{i}$ and $t_{*}\left(R^{2}\right)=$ $-(1 / l) q^{*} \psi_{1}-(1 / 2) q^{*} \sum_{i=2}^{b} \psi_{i}$. Indeed, from $f^{*} S_{b+1}^{\{1, b+1\}}=l R_{1}+A_{1}$ with $A_{1} \cdot R_{1}=0$ we deduce

$$
R_{1}^{2}=\frac{1}{l}\left(f^{*} S_{b+1}^{\{1, b+1\}}-A_{1}\right) \cdot R_{1}=\frac{1}{l} f^{*} S_{b+1}^{\{1, b+1\}} \cdot R_{1},
$$

while for $i=2, \ldots, b$ we find as above $R_{i}^{2}=\frac{1}{2} f^{*} S_{b+1}^{\{i, b+1\}} \cdot R_{i}$, hence

$$
t_{*}\left(R^{2}\right)=\sum_{i=1}^{b} t_{*}\left(R_{i}^{2}\right)=-\frac{1}{l} \psi_{1}-\frac{1}{2} \sum_{i=2}^{b} \psi_{i} .
$$

Then we get as formula for $t_{*}\left[\omega_{t}^{2}\right]$

$$
t_{*}\left[\omega_{t}^{2}\right]=-d \sum_{j=2}^{[b / 2]} q^{*} T_{b}^{j}+\frac{(2 l+1)(l-2)}{2 l} \psi_{1}+\frac{3}{2} \psi .
$$

We now substitute again the formula (4) and work out the pullback of $\psi_{1}$ that gives us the extra term in the formula for $\lambda$. This completes the proof of Theorem 1.2 . 


\section{REFERENCES}

[1] G. Farkas, A. Gibney: The Mori cone of moduli spaces of pointed curves of small genus Trans. A.M.S. 355 (2002), 1183-1199.

[2] G. van der Geer, A. Kouvidakis: The class of a Hurwitz divisor on the moduli of curves of even genus. arXiv: 1005.0969v2.

[3] J. Harris: On the Kodaira dimension of the moduli space of curves, II. The even genus case. Invent. Math. 75 (1984), p. 437-466.

[4] J. Harris, I. Morrison: Moduli of Curves. Graduate Texts in Mathematics 187, 1998, Springer.

[5] J. Harris, D. Mumford: On the Kodaira dimension of the moduli space of curves. Inventiones Mathematicae 67, (1982), 23-86.

[6] S. Keel: Intersection theory of moduli space of stable N-pointed curves of genus zero. Trans. A.M.S. 330, 1992, 545-574.

[7] A. Kokotov, D. Korotkin, P. Zograf: Isomonodromic tau function on the space of admissible covers. arXiv:0912.3909v3.

[8] D. Mumford: Stability of projective varieties. L'Enseignement Mathématique XXIII (1977), p. 39-110.

Gerard van der Geer

Korteweg-de Vries Instituut, Universiteit van Amsterdam, Postbus 94248, 1090 GE Amsterdam, The Netherlands

E-mail: G.B.M.vanderGeer@uva.nl

Alexis Kouvidakis

Department of Mathematics, University of Crete,

GR-71409 Heraklion, Greece

E-mail: kouvid@math.uoc.gr 